\title{
RNA sequencing as a tool for understanding biological complexity of abiotic stress in plants
}

\author{
Vedpal Singh Malik ${ }^{1}$
}

Received: 23 June 2015 / Accepted: 23 August 2015 / Published online: 17 October 2015

(C) Society for Plant Biochemistry and Biotechnology 2015

The radical innovations, reduction in sequencing cost and speed is transforming plant research. Genomes of many plants are completely sequenced and many genome sequences are on the way. Plant scientists use state of the art sequencing tools to aid crop improvement by understanding plant Biochemistry and physiology. Instead of backbreaking purification and characterization of proteins, characterization of Cellular RNA transcripts is a gateway to get at hard to do plant biochemistry (Martin et al. 2013, Kellner et al. 2015).

The complete set of transcripts in a cell, both in relations of type and quantity are called as Transcriptome. The arrival of high-throughput sequencing based methods has placed the study of transcriptomes within reach. This involves direct sequencing of complementary DNAs (cDNAs) using high-throughput DNA sequencing technologies (Severin 2010).

The tools and technology for RNA sequencing (RNA-Seq) and transcriptome exploration are promptly moving ahead (Martin et al. 2013).The knowledge so gained could assist in appreciating the importance of erratic and altered expression of genes as they endorse complex traits such as abiotic stresses. Enquiry into genome-wide differential RNA expression under varying environments may yield intuitions into

Vedpal Singh Malik

vedpalm@gmail.com

1 USDA, 4700 River Road, Riverdale, MD 20737, USA biological corridors, complexity and molecular mechanisms that police activity of genes involved in adaptation to abiotic stresses (Bhardwaj et al. 2015, Van Verk et al. 2013, Voytas 2013; Dolgin (2015). The technology may be used for detecting cellular pathway alterations and differential gene expression during abiotic stresses. RNA-seq may yield information about the affiliations between genes and their products and allow isolation of genes for traits whose biochemistry is difficult. Genes involved in abiotic stress such as drought tolerance in weeds whose genomes are not yet sequenced may be defined (5). Such genes may be codon optimized, chemically synthesized and expressed in crop plants to understand their impact on the overall physiological fitness of the transgenic plant (Kellner et al. 2015, Góngora-Castillo et al. 2012, Malik 2013). The synthetic genes can also be expressed in Phichia Pastoris or Escherichia coli to produce corresponding protein for biochemical studies. This technology could illuminate structural and regulatory gene networks to enlighten how plants reply to fast evolving hints and their environs (González-Ballester et al. 2010).

Fast analyses of plant's fluctuating transcriptomes through sequencing of their related complementary DNA populations allowing sequencing of the RNA transcripts in a cell including mRNA transcripts, small RNA, miRNA, tRNA is referred to as RNA-sequencing (RNA-seq). It can top score unique RNA existence and quantity from novel genomes of ferns, mosses, algae, weeds and other hardy plants adapted to stress at a precise point of development and physiological stage. Analysis of genome-wide differential RNA expression provides canvassers with greater insights into biological pathways and molecular mechanisms that regulate cell fate, development, and changes during and due to abiotic stresses 
(Duhoux et al. 2015). For mRNA analysis, the 3' polyadenylated (poly(A) tail is hybridized with poly (T) oligos that are linked to magnetic beads. The non-poly(A) RNA does not bind to the beads, $90 \%$ of which is ribosomal RNA and is removed by probe hybridization. Remaining RNA is non coding that is hydrolyzed into 200-300 nucleotides and the cDNA is further broken to the fragment length required by the sequencing system. The RNA is fractionated on size exclusion gels before addition of linkers to the 3 ' and 5' end used for sequencing.

Bioinformatics tool can then be used to analyze these sequences to stare at alternative gene spliced transcripts, posttranscriptional modifications, gene fusion, exon/intron boundaries, transcription start sites, gene boundaries, new splicing variants, and insight of the variable level of gene expression (3). RNA-seq avoids prejudices against low-abundance transcripts and enriched transcript termini. Analysis of the transcriptome recognized $42 \%$ of intron-containing genes of Arabidopsis and $48 \%$ of rice (Oryza sativa) genes as alternatively spliced. Observation of unbiased population of transcripts consents the documentation of novel transcripts, fusion transcripts and non- coding RNAs that go undetected with dissimilar technologies (Nagalakshmi et al. 2010).

Time course experiments involving RNA-Seq permit a farreaching gestalt and exact account of the physiological changes happening over time. Quantifying mRNA levels could tell how the transcriptional machinery of the cell is affected by the changing abiotic stresses $(4, \& 7)$. Post transcriptional gene regulation by RNA interference may intrude the correlation between the abundance of mRNA and the related proteins. RNA-seq is limited to transcribed regions. Differential regulation of the splice isoforms of the same gene may be marked to foretell their biological functions (9). The deep sampling used in RNA-seq may slice up the genetic substructures of precise phenotypes while searching for complex interactions within polyploid genomes (Ilut et al. 2012).

The study of transcriptome assemblies reduces the intricacies of ploidy, genome sizes with repetitive sequences, entire or partial genome duplication, heterozygosity and paralogy. It allows access of the gene space of unsequenced recalcitrant species. Technological perfections in sequencing platforms; software and smart algorithms will further improve management and mining of large, repetitive plant genomes leading to understanding of plant biochemistry and physiology for application to crop improvement (Lohse et al. 2012, Moerkercke et al. 2013).

Declaration of conflict of interest The author declares that he has no conflict of interest.

\section{References:}

Bhardwaj AK, Joshi G, Kukreja B, Malik V, Arora P, Pandey R, Shukla RN, Bankar KG, Katiyar-Agarwal S, Goel S, Jagannath A, Kumar A, Agarwal M (2015) Global insights into high temperature and drought stress regulated genes by RNA-Seq in economically important oilseed crop Brassica juncea. BMC Plant Biology 15:9. doi:10. 1186/s12870-014-0405-1

Dolgin E (2015) Elaborate architecture of RNA. Nature 523:398-399

Duhoux A, Carrère S, Gouzy J, Bonin L, Délye C (2015) RNA-Seq analysis of rye-grass transcriptomic response to an herbicide inhibiting acetolactate-synthase identifies transcripts linked to nontarget-site-based resistance. Plant Mol Biol 87(4-5):473-487. doi: 10.1007/s11103-015-0292-3 Epub 2015 Jan 31

Góngora-Castillo E, Fedewa G, Yeo Y, Chappell J, DellaPenna D, Buell CR (2012) Genomic approaches for interrogating the biochemistry of medicinal plant species. Methods Enzymol 517:139-159

Ilut DC, Coate JE, Luciano AK, Owens TG, May GD, Farmer A, Doyle JJ (2012) A comparative transcriptomic study of an allotetraploid and its diploid progenitors illustrates the unique advantages and challenges of RNA-seq in plant species. Am J Bot 99(2):383-396

Kellner F, Kim J, Clavijo BJ, Hamilton JP, Childs KL, Vaillancourt B, Cepela J, Habermann M, Steuernagel B, Clissold L, McLay K, Buell CR, O'Connor SE (2015) Genome-guided investigation of plant natural product biosynthesis. The Plant Journal 82:680-692. doi: $10.1111 /$ tpj. 12827

González-Ballester DG, Casero D, Cokus S, Pellegrini M, Merchant SS, Grossman AR (2010). RNA-Seq Analysis of Sulfur-Deprived Chlamydomonas Cells Reveals Aspects of Acclimation Critical for Cell Survival. The Plant Cell, 22: 2058-2084, June 2010, www. plantcell.org. American Society of Plant Biologists

Lohse M, Bolger AM, Nagel A, Fernie AR, Lunn JE, Stitt M, Usadel B (2012) RobiNA: a user-friendly, integrated software solution for RNA-Seq-based transcriptomics. Nucleic Acids Res 2012:1-6. doi:10.1093/nar/gks540

Moerkercke A, Fabris M, Pollier J, Baart G, Rombauts S, Hasnain G, Rischer H, Memelink J, Oksman-Caldentey K, Goossens A (2013) CathaCyc, a metabolic pathway database built from catharanthus roseus RNA-Seq data. Plant Cell Physiol (2013) 54(5):673-685. doi:10.1093/pcp/pct039

Malik VS (2013) The new technologies offer possibilities for targeted genome engineering. Ind J Plant Physiol 18:95-97

Martin L, Fei Z, Giovannoni J, Rose JK. (2013) Catalyzing Plant Science Research with RNA-seq. Front Plant Sci 4:66. http://www.ncbi.nlm. nih.gov/pmc/articles/PMC3899965/

Nagalakshmi U, Waern K, Snyder M (2010) RNA-Seq: A Method for Comprehensive Transcriptome Analysis. Current Protocols in Molecular Biology:4.11.1-4.11.13 January 2010. Published online January 2010 in Wiley Interscience (www.interscience.wiley.com). doi:10.1002/0471142727.mb0411s89

Severin AJ (2010) Woody JL, Tsi Bolon Y, joseph B, diers BW, farmer AW, muehlbauer GJ, nelson RT, grant D, Specht JE, graham MA, cannon SB, may GD, Vance CP, shoemaker RC (2010) RNA-Seq atlas of Glycine max: a guide to the soybean transcriptome. BMC Plant Biology 10:160. doi:10.1186/1471-2229-10-160

Van Verk MC, Hickman R, Corné MJ, Saskia P, Van Wees CM (2013) RNA-Seq: revelation of the messengers. Trends Plant Sci 18(4): 175-179

Voytas DF (2013) Plant genome engineering with sequence-specific nucleases. Annu Rev Plant Biol 64:327-504 